\title{
Cuidado, valores y género: la distribución de roles familiares en el imaginario colectivo de la sociedad española
}

\author{
Care, values and gender: the distribution of family roles \\ in the collective imagination of Spanish society
}

\author{
Iratxe Aristegui Fradua, Usue Beloki Marañon, Raquel Royo \\ Prieto, María Silvestre Cabrera • iariste@deusto, usue.beloki@ \\ deusto.es, raquel.royo@deusto.es, maria.silvestre@deusto.es \\ EQUIPO DE INVESTIGACIÓN DEUSTO VALORES SOCIALES. \\ DEPARTAMENTO DE TRABAJO SOCIAL Y SOCIOLOGÍA. \\ UNIVERSIDAD DE DEUSTO
}

Recibido: $25 / 11 / 2018$

Aceptado: 03/12/2018

\section{Resumen}

El Equipo Deusto Valores Sociales, al que pertenecen las autoras de este texto, representa a España en la EVS y ha sido la encargada de aplicar la encuesta también en su última edición 2018 (trabajo de campo entre diciembre de 2017 y enero de 2018), para lo que ha contado con la financiación otorgada al proyecto Cambio de valores en España y en Europa: identidad europea, justicia social y solidaridad ante nuevos escenarios (Ref.: CSO2016-77057-R) en el Programa Estatal de Investigación, Desarrollo e Innovación Orientada a los Retos de la Sociedad del Ministerio de Economía y Competitividad en la convocatoria de 2016.

Este artículo analiza de forma descriptiva algunos valores, percepciones y actitudes de la sociedad española a partir de la Encuesta Europea de Valores (EVS) en su aplicación en España en 2018. En este caso, nos hemos centrado en el estudio de los valores asociados con las tareas del cuidado, tanto de la prole como de las personas ascendientes. El principal objetivo de este trabajo ha sido analizar la convivencia de nuevos roles laborales con tradicionales roles domésticos asociados a las mujeres. También se ha estudiado si la sociedad, a nivel valorativo y discursivo, está construyendo nuevas formas de asumir las tareas de cuidado. En este sentido, podemos afirmar que hemos encontrado una distancia entre la práctica del cuidado y la concepción de la responsabilidad del cuidado.

Palabras clave: Mujeres, cuidado, valores, roles de género, conciliación corresponsable.

\section{Abstract}

Deusto Social Values Team, to which the authors of this text belong, represents Spain in the EVS and has been commissioned to apply the survey also in its latest edition 2018 (between December 2017 and January 2018). In order to aim this, it has counted on the financing granted to the project Change of values in Spain and in Europe: European identity, social justice and solidarity before new scenarios (Ref .: CSO2016-77057-R) in the State Program of Research, Development and Innovation Oriented to the Challenges of the Society of the Ministry of Economy and Competitiveness in 2016.

This article analyzes descriptively some values, perceptions and attitudes of Spanish society from the European Values Survey (EVS) in its application in Spain in 2018. In this case, we have focused on the study of the values associated with the tasks of care, both of the offspring and of the ascendants. The main objective of this work has been to analyze the coexistence of new labor roles with traditional domestic roles associated with women. It has also been studied whether society, at a value and discursive level, is building new ways of as- 
suming care tasks. In this sense, we can affirm that we have found a distance between the practice of care and the conception of the responsibility of care.

Keywords: Women, care, values, gender roles, conciliation.

\section{REVISIÓN DE LA LITERATURA. CUIDAR Y SOSTENER LA VIDA: ¿UN TRABAJO DE MUJERES?}

En las últimas décadas, la sociedad occidental ha experimentado profundos cambios en el estatus de las mujeres. En particular, la incorporación masiva de las mujeres al empleo altera sustancialmente las bases de la familia tradicional y da paso a la postmoderna en la que se difumina la rígida división de roles de género tradicionales (Meil, 1999).

Sin embargo, investigaciones recientes sobre la división de roles familiares muestran que las mujeres siguen asumiendo la mayor parte del trabajo doméstico y de cuidados (INE, 2018). Según la encuesta de calidad de vida de 2016, en la Unión Europea, el 92\% de las mujeres de 25 a 49 años cuida a sus hijos o hijas diariamente, frente al 68\% de los hombres de la misma edad. En el Estado español esta distancia es mayor (95\% vs. 68\%), siendo el séptimo de los veintiocho países de la Unión Europea con mayor brecha de género, por detrás de Grecia, Malta, Rumanía, Eslovaquia, República Checa y Polonia (INE y EUROSTAT, 2017). En lo que se refiere al cuidado de personas mayores de 75 años con enfermedad o discapacidad, el porcentaje de mujeres mayores de 18 años que realiza esta tarea al menos varias veces por semana duplica al de los hombres (10\% vs. 5\%), siendo también superior que en el entorno europeo ( $8 \%$ vs. 5\%).

Tabla 1. Horas semanales dedicadas a actividades de cuidados y tareas del horal ${ }^{(1)}$. España y UE-28. 2016

\begin{tabular}{|l|c|c|c|c|}
\hline \multirow{2}{*}{} & \multicolumn{2}{|c|}{ España } & \multicolumn{2}{c|}{ UE-28 } \\
\cline { 2 - 5 } & Mujeres & Hombres & Mujeres & Hombres \\
\hline Cuidado o educación de hijos/as & 38 & 23 & 38 & 21 \\
\hline Cuidado o educación de nietos/as & 16 & 16 & 16 & 12 \\
\hline Cocinar o hacer laborales domésticas & 20 & 11 & - & - \\
\hline $\begin{array}{l}\text { Cuidado de familiares, vecinos o amigos enfermos con } \\
\text { discapacidad menores de 75 años }\end{array}$ & 20 & 14 & 16 & 13 \\
\hline $\begin{array}{l}\text { Cuidado de familiares, vecinos o amigos enfermos o } \\
\text { con discapacidad mayores de 75 años }\end{array}$ & 18 & 14 & 12 & 10 \\
\hline
\end{tabular}

(1) Personas de 18 y más años.

Fuente: encuesta de realidad de vida 2016 (Eurofound en INE, 2018).

La encuesta mencionada también evidencia que en el Estado español las mujeres mayores de 18 años dedican más horas semanales que los hombres a las actividades de cuidado o educación de la prole (15 horas más), cocinar o hacer labores domésticas (9 horas más) y al 
cuidado de personas con enfermedad o discapacidad (en torno a 5 horas más). El cuidado de nietos y nietas constituye la única actividad considerada en la que los datos reflejan una distribución simétrica en términos del porcentaje de mujeres y hombres que realizan la actividad (en torno al 32\%) y del tiempo invertido en ella (16 horas) (INE, 2018).

Asimismo, según la Encuesta Nacional de Condiciones de Trabajo de 2015, el análisis conjunto del trabajo remunerado y no remunerado en el Estado español muestra que las jornadas de trabajo de las mujeres son más largas que las de los hombres. Las primeras afrontan jornadas semanales de 63,6 horas (dedicando 34,2 horas al trabajo remunerado; 26,5 horas al no remunerado y 2,9 horas a los desplazamientos) frente a las jornadas de 56,7 horas semanales de los segundos (que emplean en el trabajo remunerado, no remunerado y desplazamientos 39,9; 14 y 2,8 horas, respectivamente) (Instituto Nacional de Seguridad e Higiene en el Trabajo, 2017: 88). Cabe señalar que los hombres dedican habitualmente el mismo número de horas al trabajo no remunerado (14 horas a la semana) con independencia de que su empleo sea a tiempo parcial o a jornada completa. Sin embargo, las mujeres aumentan el tiempo dedicado a este tipo de trabajo (30 horas a la semana) cuando su empleo es a tiempo parcial (Instituto Nacional de Seguridad e Higiene en el Trabajo, 2017:88).

En definitiva, los datos dibujan un panorama aún asimétrico que se caracteriza por una mayor dedicación de las mujeres a los trabajos domésticos y de cuidado, una mayor dedicación de los hombres al empleo y una carga total de trabajo mayor para ellas. Estas importantes diferencias en el modo de organizar los trabajos y el tiempo en la sociedad española y en el entorno europeo, tienen implicaciones en la calidad de vida y en las posibilidades de desarrollo personal de mujeres y varones.

El olvido de la aportación social y económica de las personas cuidadoras, que como hemos visto son predominantemente mujeres, tiene además importantes consecuencias en el diseño de las políticas públicas y en la —desigual—distribución del reconocimiento y los beneficios sociales, tal y como señalan Folbre y Bittman:

El trabajo de cuidar a las personas dependientes tiene consecuencias positivas para la sociedad en su conjunto (...). Sin embargo, los individuos (...) que realizan importantes aportaciones a la familia y al trabajo comunitario generalmente pagan una gran multa económica y social. (Folbre y Bittman, 2004: 1)

Como bien señalan Díaz y Simó (2016), la escasa consideración social de los trabajos de cuidado y su feminización son la primera forma de discriminación de género y sobre la que se construyen otras discriminaciones como la salarial.

\section{Bases simbólicas de la feminización del cuidado}

La existencia humana y social no sólo depende de la producción material, sino también de múltiples actividades rutinarias que proporcionan alimento, vestido, protección y cuidado. 
Este trabajo, desempeñado generalmente en el marco de las familias, es tan importante para el bienestar y para la supervivencia de una sociedad como el trabajo remunerado de la economía de mercado. Sin embargo, con frecuencia permanece ausente en los análisis económicos y se trivializa en la vida cotidiana, en parte porque se considera un «trabajo de mujeres» (Coltrane, 2000: 1208-1209), invisible y desprovisto de la consideración de auténtico trabajo (Giddens, 2002: 507-508), y constituye una actividad que se encuentra entre los niveles más bajos de aprecio social.

Según M. a Ángeles Durán, uno de los argumentos más empleados para restar valor y para ignorar la condición económica del trabajo familiar es precisamente negarle su carácter de trabajo (Durán, 1978: 23). El hecho de que un trabajo imprescindible para la vida ni siquiera se identifique como trabajo no es en modo alguno casual. Los conceptos que utilizamos en la vida cotidiana - y en el ámbito científico- no son meras concepciones neutras que reflejan la realidad como si de un espejo se tratase, sino que son constructos sociales. Así, para abordar el estudio de ámbitos tan naturalizados como el trabajo familiar es importante comprender que los conceptos a los que recurrimos son en sí mismos construcciones culturales e ideológicas, atravesadas por el poder y productos de un determinado desarrollo histórico ${ }^{1}$.

Un elemento central que subyace a la feminización del cuidado - y al escaso valor atribuido al mismo - es lo que se conoce como la división tradicional del trabajo, según la cual a las mujeres se les atribuye el trabajo reproductivo, es decir, el trabajo doméstico y de cuidados, mientras que a los hombres les corresponde la provisión económica de la familia y el ejercicio de la autoridad. Este modelo, al que solemos denominar «la familia tradicional», no constituye una categoría universal y transhistórica, sino que alude a una forma específica y unívoca de relaciones de género que se impuso en la última fase de la industrialización (Scott y Tilly, 1984: 58). Así, de acuerdo con el ideal victoriano de las esferas separadas, el mundo exterior es «masculino» y el doméstico, «femenino»; tal y como corresponde a las características esencialmente distintas, contrapuestas y complementarias que se suponen en mujeres y hombres. En este esquema, la mujer, esposa y madre, es el ángel del hogar, el eje de la familia y la guardiana de las buenas costumbres; en definitiva, un ser doméstico, delicado, dependiente de la protección de un padre o un marido (McDougall, 1984: 91), siendo su impulso a la maternidad análogo al impulso sexual del varón.

Aunque nuestra concepción cultural de la maternidad tiene una larga historia, está vinculada especialmente a este discurso victoriano burgués sobre la mujer y a esta división sexual de esferas que materialmente fundamentó el aislamiento madre-hijo/a y nos legó la imagen de la madre ideal que garantizaría tanto una descendencia moralmente perfecta como un mundo moralmente deseable (Chorodow y Contratto, 1982: 63-64).

\footnotetext{
${ }^{1}$ Para una revisión más amplia del surgimiento de la dicotomía público-doméstico y del concepto de trabajo, así como de los significados culturales de la maternidad y la paternidad, véase Royo (2018). Maternidad, paternidad y conciliación en la CAE. ¿Es el trabajo familiar un trabajo de mujeres?, Bilbao: Universidad de Deusto.
} 
Como consecuencia de este desarrollo histórico, la imagen occidental de la maternidad aparece marcada por la mitificación de la relación íntima entre la madre y el hijo o hija². Patrice Di Quinzio (1999) se refiere a la «maternidad esencial», que requiere la atención exclusiva y desinteresada de la madre, basándose en las características psicológicas femeninas de empatía, reconocimiento de las necesidades de las demás personas y autosacrificio. Dichas características se suponen naturales en las mujeres, por lo que se presume que todas ellas desean la maternidad, que es necesaria e inevitable para su desarrollo y satisfacción emocional. La maternidad así construida sustenta una serie de estereotipos sobre la «buena» y la «mala» madre. Para Swigart (1991), la «buena madre» es una mujer que sólo quiere lo mejor para su prole y que intuye sus necesidades sin esfuerzo alguno. Inmune al aburrimiento, vive la crianza como una fuente de placer que no requiere sacrificio $^{3}$. Por el contrario, la «mala madre» es una mujer que se aburre con su prole, que es narcisista, que carece de empatía y que está centrada en sus propios intereses y problemas. Por ello, es insensible a las necesidades de sus hijos e hijas, que acaban padeciendo trastornos psicológicos de los que la madre no es consciente. En definitiva, una madre que no sigue el patrón establecido es una madre desnaturalizada, un monstruo (Donapetry, 2002: 52$)^{4}$.

Los estereotipos anteriores, además de no sustentarse en datos empíricos, afectan negativamente a la imagen de las mujeres sin dañar la de los hombres. Esto ocurre porque en el imaginario colectivo, la maternidad está fuertemente vinculada a la identidad femenina. A través de los siglos, el patriarcado se ha servido de la capacidad biológica de las mujeres para la reproducción para fundamentar la unidimensionalidad del ser femenino y la permanente identificación entre feminidad y maternidad (Cid, 2002: 11). De hecho, las características psicológicas positivas atribuidas a las mujeres en nuestra cultura - afectuosas, sensibles a las necesidades de las demás personas, amables, comprensivas...- coinciden con lo que socialmente se espera de una madre.

En la concepción tradicional, los hombres, por su parte, «son quienes trabajan» para sostener a la familia ${ }^{5}$, de forma que la imagen de la paternidad tradicional en el contexto occidental se ha asociado tradicionalmente a la función de la provisión económica - breadwinner-, que implica normalmente la ausencia del hogar familiar durante gran parte del día, lo que marca una relación normalmente más distante o, al menos, menos frecuente que la

\footnotetext{
${ }^{2}$ Conviene precisar que vamos a referirnos a la «maternidad» y la «paternidad» como instituciones, no a las experiencias de los individuos concretos que, aun modeladas por la institución, son, lógicamente, diversas.

${ }^{3}$ En contraste con estas imágenes normativas, caracterizadas por las idealizadas y poco realistas expectativas hacia las madres (Coltrane, 1998: 81), los comportamientos de las madres están fuertemente modelados por el contexto social, histórico, económico y político.

${ }^{4}$ Asimismo, el estereotipo de las mujeres que deciden no ser madres describe a mujeres anormales,egoístas, inmorales, irresponsables, inmaduras, no felices, no realizadas y no femeninas (Osborne, 1993: 139).

${ }^{5}$ Aunque numerosas mujeres de clase baja continuaron trabajando para garantizar su supervivencia y la de sus familias — siendo fundamental su contribución económica一, su papel principal socialmente definido se circunscribía a las funciones propias de su sexo. En este sentido, conviene destacar que, a pesar de que la doctrina de las esferas separadas caló en la clase baja, lo hizo más como un ideal o una aspiración que como una realidad (Coontz, 2005: 168-169), ya que estas mujeres traspasaban la frontera entre la esfera pública y privada.
} 
que la madre mantiene con la prole (Mander, 2004: 67). Por tanto, el rol paterno en el cuidado de su descendencia se entiende como esencialmente indirecto, mediante la función primaria de proveedor familiar y de apoyo emocional para la madre (Barnett y Baruch, 1988: 66$)^{6}$.

Además, durante la industrialización no sólo se configura la dicotomía entre el ámbito público y el doméstico a la que nos hemos referido, sino que se gesta el concepto de trabajo equiparándolo y reduciéndolo al empleo (Carrasco y Mayordomo, 2000: 102) —es decir, a la actividad pública, definida, considerada socialmente útil, que obtiene una remuneración medible y optimizable (Gorz, 1997: 26, 36)—. Tal y como señala Gorz:

Lo que nosotros llamamos trabajo es una invención de la modernidad. La forma en que lo conocemos, lo practicamos y lo situamos en el centro de la vida individual y social fue inventada y luego generalizada con el industrialismo. (Gorz, 1997: 25)

De esta forma, el hogar y la maternidad se configuran como referente fundamental de la feminidad $-\mathrm{y}$ el trabajo doméstico como un «no-trabajo»-, mientras el trabajo definido como empleo adquiere una posición central en el orden social y en la identidad de la mayoría de los hombres, a la vez que el lugar en el que trabajan se convierte en el ámbito más relevante para la integración social.

La Economía convencional asumió y contribuyó a estos postulados, dejando fuera del análisis económico las actividades no mercantiles, su articulación con la economía y su función de sustento del sistema social (Todaro y Rodríguez, 2001: 10). Sin embargo, el trabajo familiar es una condición imprescindible para que el mercado exista (Dones i Treballs, 2003: 18). Los sistemas económicos, aun presentándose como autónomos, siempre han dependido de una actividad doméstica que reproduce la vida y la fuerza de trabajo sin coste alguno para dichos sistema, pero en particular en el capitalismo esta relación permanece oculta para asegurar el desplazamiento de costes desde la producción capitalista hacia la esfera doméstica (Carrasco, 2003: 34).

En la actualidad la doctrina de las esferas separadas está puesta en cuestión por la incorporación masiva de las mujeres al ámbito laboral, pero para Coltrane (1996) pervive en el mundo de las creencias y valores socialmente compartidos donde los diversos roles, aptitudes y espacios se atribuyen a hombres y a mujeres. Más que cualquier otra creencia, la asignación de los espacios doméstico y público a unas y a otros está íntimamente vinculada a las identidades de género, es decir, a lo que significa ser una mujer o un hombreen nuestra sociedad ${ }^{7}$. En palabras de Coltrane:

\footnotetext{
${ }^{6}$ Además, la percepción de la paternidad tradicional remite a una figura autoritaria y disciplinadora (Moss, 1995: XI).

${ }^{7}$ Lagarde afirma que el mandato cultural impele a las mujeres a convertirse en un ser-para-los-otros —en el centro de su vida están las demás personas y sus necesidades, no ella misma-, mientras que los varones se construyen como seres-para-sí-en-el-mundo — se tienen a sí mismos como objetivo de sus energías vitales, como su centro- (Lagarde, 1996: 60-62).
} 
Se espera que las mujeres amables y delicadas permanezcan en el hogar para cuidar a la prole y a la familia, permitiendo a los hombres valientes y agresivos aventurarse en los mundos competitivos del trabajo, la política y la guerra. (Coltrane, 1996: 25)

Desde estos postulados, diversas investigaciones sugieren que mujeres y hombres desarrollan distintas tareas porque sus prácticas afirman y reproducen sus identidades de género (Coltrane, 2000: 1213) y que las ideologías que rodean el trabajo familiar constituyen en sí mismas un ámbito que refleja la reproducción del género (Erickson, 2005: 340).

Ahora bien, en un mundo transformado por el cambio del estatus femenino, en el que una imparable retórica sobre la igualdad inunda el ámbito político y en el que surgen modelos de paternidad y maternidad más igualitarios — nuevas paternidades ${ }^{8}$ (Parke, 2000; Peterson y Steinmetz, 2000) y maternidades menos intensivas-, cabe preguntarse de qué manera estas concepciones heredadas del pasado perviven o se transforman en el imaginario colectivo y cuál es su extensión en los distintos grupos sociales.

\section{METOdOLOGÍA}

El diseño metodológico es cuantitativo y toma como referencias los resultados obtenidos de la aplicación de la Encuesta Europea de Valores en 2018, a una muestra representativa de la población española mayor de 18 años. El trabajo de campo se realizó en enero de 2018 a cargo del Equipo Deusto Valores Sociales de la Universidad de Deusto que representa a España en este consorcio internacional. La muestra total con la que se ha trabajado es de $\mathrm{N}=1212$. La población objetivo consistía en todas las personas mayores de 18 años residentes en hogares privados en España independientemente de su nacionalidad, ciudadanía o idioma. Por lo tanto, la población objetivo incluía a todos los residentes (hogares privados) en España nacidos antes o en septiembre de 1999.

La muestra se elaboró en tres etapas. En primer lugar, se seleccionaron secciones censales municipales mediante un procedimiento aleatorio simple con probabilidad proporcional a la población objetivo en cada región (comunidades autónomas). En una segunda etapa, se seleccionaron 4331 hogares de manera proporcional a la población objetivo de las secciones del censo de los municipios. Finalmente, la tercera etapa (en el campo propiamente dicho) se seleccionó a una persona de cada hogar muestreado utilizando el método Kish. El posterior análisis de datos se ha llevado a cabo a través del programa estadístico SPSS (versión 21). Al tratarse de una aproximación descriptiva, los resultados se basan principalmente en porcentajes y en la aplicación del estadístico Chi-cuadrado.

\footnotetext{
${ }^{8}$ En este sentido, según Alberdi (1999: 153), frente al modelo tradicional de mitificación de la maternidad, surge actualmente un modelo alternativo de padre que se presenta como ejemplar o deseable y que prescribe una mayor presencia paterna en la vida de la prole y la participación equilibrada de la pareja en el cuidado de los hijos e hijas. En definitiva, una idea de paternidad más orientada a compartir el cariño y el cuidado que las ideas tradicionales de provisión económica y autoridad.
} 


\section{RESULTADOS}

Los resultados que presentamos a continuación se han dividido en dos grandes apartados. El primero de ellos gira en torno al empleo femenino y la familia tradicional, en un contexto en el que se superponen nuevos roles laborales y viejos roles domésticos (Tobío, 2005), y explora en el imaginario colectivolas implicaciones familiares que se perciben cuando las mujeres trabajan fuera del hogar. El segundo apartado nos sitúa ante un escenario marcado por el envejecimiento poblacional e indaga en el posicionamiento de la ciudadanía española en torno al deber de cuidado ascendente.

\section{a) El trabajo de las mujeres: nuevos roles laborales versus viejos roles domésticos}

En las últimas décadas, el empleo femenino ha pasado a formar parte de la experiencia cotidiana de un número creciente de mujeres, lo que introduce un elemento de ruptura con el modelo de familia tradicional, en la medida que el trabajo remunerado femenino altera las bases del contrato social tradicional (Alberdi, 1999:254). Ahora bien, ¿en qué medida este cambio vinculado, entre otros factores, a las nuevas demandas socioeconómicas — del mercado laboral, de las economías familiares - supone asimismo una transformación simbólica de los valores tradicionales asociados a la división sexual del trabajo y a la maternidad?

Varias preguntas de la encuesta europea de valores nos permiten explorar estas cuestiones en el imaginario colectivo de la sociedad española. Abordamos en primer lugar los valores e imágenes actuales sobre cómo debe realizarse la distribución del trabajo productivo y reproductivo en el ámbito doméstico, es decir, sobre quién debe realizar la función de proveer económicamente a la familia, y sobre quién debe asumir el cuidado del hogar y sus miembros.

Según este estudio, más de ocho de cada diez personas en el Estado español (86,3\%) se posicionan en contra del discurso tradicional de género sobre la familia, que como hemos visto atribuye a los hombres el papel de «breadwinner» y asigna a las mujeres el cuidado en el ámbito doméstico-familiar. La extensión de esta opinión, que podría relacionarse con el auge del principio de igualdad en nuestra sociedad (Castells, 1997: 160), es claramente perceptible en los distintos segmentos sociales definidos en la tabla 2.

Sin embargo, la discrepancia con la división tradicional de roles familiares no se distribuye de forma homogénea entre la población, existiendo diferencias significativas en función del género y la edad. Como se observa en dicha tabla, el desacuerdo con los postulados tradicionales es particularmente alto en las personas menores de 55 años, mientras que, en los siguientes tramos de edad, sobre todo entre las personas mayores de 65, la oposición a la división tradicional, disminuye (73,3\%). En lo que se refiere al género, las mujeres manifiestan niveles de desacuerdo algo mayores que los de los hombres, lo que podría 
Cuidado, valores y género | I. Aristegui Fradua, U. Beloki Marañon, R. Royo Prieto, M.a Silvestre Cabrera

relacionarse con el mayor y más rápido cambio que las primeras han experimentado en sus roles en comparación con los segundos (Hochschild y Machung, 2003: 214).

Tabla 2. Grado de acuerdo* con la afirmación «El trabajo del hombre es ganar dinero, el trabajo de la mujer es cuidar del hogar y la familia» (\% horizontales). España. 2018

\begin{tabular}{|c|c|c|c|}
\hline & $\begin{array}{c}\text { Acuerdo } \\
\text { (muy acuerdo + acuerdo) }\end{array}$ & $\begin{array}{r}\text { Desacuerdo } \\
\text { (desacuerdo + muy desacuerdo) }\end{array}$ & NS/NC \\
\hline TOTAL & 12,7 & 86,3 & 1,0 \\
\hline Sexo & & & 1,4 \\
\hline Mujer & 11,0 & 87,6 & 0,6 \\
\hline Hombre & 14,8 & 84,6 & 0,1 \\
\hline Edad & & & 1,2 \\
\hline $18-24$ & 7,8 & 92,1 & 1,7 \\
\hline $25-34$ & 7,8 & 91,0 & 0,4 \\
\hline $35-44$ & 8,2 & 90,1 & 0,6 \\
\hline $45-54$ & 6,7 & 92,9 & 1,4 \\
\hline $55-64$ & 12,9 & 86,5 & 73,3 \\
\hline 65 y & 25,3 & & \\
\hline
\end{tabular}

* En una escala del 1 al 4 siendo el 1 «muy de acuerdo», 2 «de acuerdo», 3 «en desacuerdo» y 4 «muy en desacuerdo».

Fuente: elaboración propia a partir de la base de datos de la EVS 2018. Los \% que faltan para llegar al 100\%, corresponden al ns/nc.

Esta amplia oposición a la rígida división de roles maritales no implica un apoyo total al empleo femenino, al menos en términos similares a la experiencia laboral masculina mayoritaria. Así, llama la atención la diferencia de dieciséis puntos porcentuales que existe entre la población que rechaza la división tradicional del trabajo familiar (86,3\%) y la que niega que la vida familiar sufre ante el empleo femenino a jornada completa $(69,9 \%$. En el mismo sentido, prácticamente tres de cada diez personas consultadas $(29,1 \%)$ afirman que la vida familiar sufre cuando la mujer tiene un empleo a tiempo completo. Esta creencia, aunque minoritaria, resulta significativa en la medida que podría estar relacionada con la pervivencia de las concepciones tradicionales sobre la feminidad y la maternidad, lo que sugiere que el referente doméstico-maternal no ha desaparecido del imaginario colectivo de la sociedad española. El impulso individualizador que han experimentado las mujeres -en particular de las generaciones más jóvenes- de la mano del empleo (Beck y BeckGernsheim, 2003), coexiste así con resistencias a la menor presencia de las mujeres en los entornos familiares.

El análisis estadístico arroja, de nuevo, diferencias significativas en función del género y la edad. Así, las mujeres son quienes manifiestan en mayor medida el menoscabo de la vida 
familiar ante el empleo femenino a tiempo completo, lo que sugiere que estas interiorizan en mayor medida que los hombres el peso de las expectativas de género con relación al rol familiar femenino. Otra lectura de este dato, no necesariamente incompatible con la anterior, podría asociarse con las condiciones estructurales vinculadas a la organización socioeconómica —desigualdades laborales, desincronización de los horarios laborales acentuada por la falta de servicios asequibles y de calidad - y/o a la falta de corresponsabilidad en los hogares. Ello, unido a las constricciones simbólicas a las que nos hemos referido, hacen que las mujeres actúen como variable de ajuste (Dones i Treballs, 2003: 24) a la hora de armonizar el ámbito familiar y laboral, en la medida en que su mayor protagonismo en la conciliación se contempla como la opción más racional y natural. Esto supone para muchas mujeres una posición sujeta, amarrada - una condición - en la que deben seguir equilibrando sus roles, adaptando sus vidas a los deseos y necesidades de las demás personas e interpretando su experiencia desde la aceptación del discurso de género (Papí y Frau, 2005: 162).

Tabla 3. Grado de acuerdo* con la afirmación «La vida familiar sufre cuando una mujer tiene un trabajo a tiempo completo» (\% horizontales) España

\begin{tabular}{|l|c|c|c|}
\hline & $\begin{array}{c}\text { Acuerdo } \\
\text { (muy acuerdo + acuerdo) }\end{array}$ & $\begin{array}{c}\text { Desacuerdo } \\
\text { (desacuerdo + muy desacuerdo) }\end{array}$ & NS/NC \\
\hline TOTAL & 29,1 & 69,9 & 1,1 \\
\hline Sexo & & & $\mathbf{1 , 2}$ \\
\hline Mujer & 29,9 & 69,0 & 0,0 \\
\hline Hombre & 27,9 & 70,9 & $\mathbf{1 , 2}$ \\
\hline Edad & & & 0,9 \\
\hline $18-24$ & 24,7 & 75,3 & 0,9 \\
\hline $25-34$ & 21,6 & 77,2 & 1,1 \\
\hline $35-44$ & 29,3 & 69,8 & 1,7 \\
\hline $45-54$ & 22,5 & 76,6 & 1,1 \\
\hline $55-64$ & 24,3 & 74,6 & 1,2 \\
\hline 65 y + & 42,9 & 55,4 & \\
\hline
\end{tabular}

* En una escala del 1 al 4 siendo el 1 «muy de acuerdo», 2 «de acuerdo», 3 «en desacuerdo»y 4 «muy en desacuerdo».

Fuente: elaboración propia a partir de la base de datos de la EVS 2018. Los \% que faltan para llegar al 100\%, corresponden al ns/nc.

En lo que se refiere a la edad, el acuerdo con las repercusiones negativas del empleo femenino en la vida familiar resulta más rotundo en el segmento de edad de mayores de 65 años, en el que más de cuatro de cada diez personas suscriben dicha opinión (42,9\%). Esto podría relacionarse con el hecho de que estas personas fueron educadas y vivenciaron un contexto en el que la feminidad estaba profundamente vinculada al ámbito domés- 
tico. Asimismo, la categoría de 35 a 44 años, que constituye el segmento que a priori está más afectado por la necesidad de compatibilizar el empleo y el cuidado de menores, apoya esta postura en mayor medida que el resto de tramos etarios menores de 65 años. Como se señalaba anteriormente, esto plantea interrogantes sobre los condicionantes contextuales, materiales y simbólicos que podrían subyacer a las dificultades para la conciliación corresponsable.

Tabla 4. Grado de acuerdo* con la afirmación «Los niños sufren con una madre trabajadora» (\% horizontales) España

\begin{tabular}{|c|c|c|c|}
\hline & $\begin{array}{c}\text { Acuerdo } \\
\text { (muy acuerdo + acuerdo) }\end{array}$ & $\begin{array}{c}\text { Desacuerdo } \\
\text { (desacuerdo + muy desacuerdo) }\end{array}$ & NS/NC \\
\hline TOTAL & 25,9 & 72,5 & 1,6 \\
\hline Sexo & & & 1,9 \\
\hline Mujer & 25,9 & 72,2 & 1,2 \\
\hline Hombre & 25,8 & 73,0 & 1,2 \\
\hline Edad & & & 1,2 \\
\hline $18-24$ & 26,9 & 71,9 & 0,9 \\
\hline $25-34$ & 18,0 & 80,8 & 0,8 \\
\hline $35-44$ & 27,5 & 71,6 & 0,5 \\
\hline $45-54$ & 17,8 & 81,4 & 4,0 \\
\hline $55-64$ & 22,7 & 76,8 & \\
\hline 65 y & 37,5 & 58,5 & \\
\hline
\end{tabular}

* En una escala del 1 al 4 siendo el 1 «muy de acuerdo», 2 «de acuerdo», 3 «en desacuerdo» y 4 «muy en desacuerdo».

Fuente: elaboración propia a partir de la base de datos de la EVS 2018. Los \% que faltan para llegar al 100, corresponden al ns/nc.

También cuando se evoca la presencia de niños o niñas emerge la resistencia social a disminuir la presencia femenina en el ámbito doméstico-familiar. Así, como se observa en la tabla 4, casi tres de cada diez personas (25,9\%) afirman que «los niños sufren con una madre trabajadora». En este caso, encontramos diferencias estadísticamente significativas en función de la edad —no así de género-. De nuevo, el segmento de edad de 35 a 44 años, que en caso de tener descendencia suele coincidir con la crianza de niños y niñas de corta edad $^{9}$, expresa un mayor apoyo a esta idea (27,5\%), congruente con los postulados de la maternidad tradicional. La persistente creencia de que las mujeres son las principales responsables del cuidado de la prole explicaría la vivencia femenina de culpabilidad, que constituye una experiencia difícil de evitar para las mujeres que trabajan fuera del hogar

\footnotetext{
${ }_{9}$ La edad media a la maternidad en el año 2017 se sitúa en 30,08 (INE, 2018).
} 
y tienen niños o niñas pequeñas (Alberdi, 1999: 168). El apoyo a esta creencia aumenta notablemente en el tramo de edad de mayores de 65 años, en el que casi cuatro de cada diez personas $(37,5 \%)$ consideran que existe sufrimiento infantil cuando la madre trabaja fuera de casa.

\section{b) El cuidado ascendente}

Nos hemos acercado a los valores y actitudes relacionados el empleo femenino durante la época de crianza. Ahora bien, no sólo necesitamos cuidados durante la infancia, sino también durante la edad adulta —y cada vez más - durante la vejez. En el Estado español la población que suscribe que Es obligación del hijo o hija proporcionar cuidado a largo plazo para los padres (52,1\%) supera a la que opina que no existe dicho deber filioparental (24,5\%). Esto sugiere la existencia de una solidaridad intergeneracional hacia la generación ascendente, que cabe relacionar con una sociedad tradicionalmente familista como la española ${ }^{10}$.

Esta prestación de cuidados a ascendientes ha sido históricamente realizada por las mujeres, por lo que resulta interesante analizar las posibles diferencias de género en la percepción de esta responsabilidad. La escasa diferencia que hemos encontrado en las posiciones de mujeres y hombres en este sentido (52,3\% versus 51,8\%) en el Estado español —que además carece de significación estadística-, como hemos visto anteriormente, no se están traduciendo en una asunción paritaria de dichas tareas ${ }^{11}$.

Tabla 5. Grado de acuerdo con la afirmación «Es obligación del hijo/a proporcionar cuidado a largo plazo para los padres, según sexo.» \% horizontales. España 2018

\begin{tabular}{|l|c|c|c|c|c|c|}
\hline & $\begin{array}{c}\text { Muy de } \\
\text { acuerdo }\end{array}$ & Acuerdo & $\begin{array}{c}\text { Ni de } \\
\text { Acuerdo/ } \\
\text { Ni en } \\
\text { Desacuerdo }\end{array}$ & Desacuerdo & $\begin{array}{c}\text { Muy en } \\
\text { Desacuerdo }\end{array}$ & NS/NC \\
\hline Mujer & 16,7 & 35,6 & 22,6 & 16,2 & 8,0 & 0,9 \\
\hline Hombre & 13,5 & 38,3 & 21,3 & 19,4 & 5,4 & 2,0 \\
\hline
\end{tabular}

Fuente: elaboración propia a partir de la base de datos de la EVS 2018.

\footnotetext{
10 Por otro lado, el hecho de que más de dos de cada diez personas (22\%) no se sitúen ni de acuerdo ni en desacuerdo con el deber filial tradicional de cuidar a padres y madres, unido al segmento de población que, como hemos visto, es contrario a dicha obligación (24,5\%), apunta a la existencia de un impulso individualizador que cuestiona este mandato tradicional en las sociedades familistas.

11 Diversos estudios muestran que el cuidado es para las mujeres cuidadoras una obligación moral y social que asumen con un cierto grado de resignación y que, en la mayoría de las ocasiones, se ejerce desde el cariño y amor que se profesa a la madre o al padre (Leonardo et al., 2007).
} 
En cambio, tal y como puede observarse en la tabla siguiente, la edad establece diferencias significativas en el posicionamiento de la sociedad española ante deber filial de cuidado ascendente, que paradójicamente suscita mayores apoyos entre los segmentos de mayor y menor edad. No resulta sorprendente el hecho de que las personas mayores de 65 años, suscriban dicha postura, ya que presumiblemente contemplan la probabilidad de requerir cuidados en un futuro cada vez menos lejano (58,1\%). En cambio, la adhesión a esta postura que encontramos en el tramo de edad más joven (62,9\%) podría responder en cierta medida a la lejanía con la que se contempla esta contingencia en esta etapa, en abierto contraste con el menor apoyo que suscita esta idea entre quienes por edad se encuentran precisamente en una situación vital que coincide con la prestación de dichos cuidados, esto es, los tramos etarios centrales (35-65 años). En ellos, tres de cada diez personas se posicionan en contra de la obligatoriedad del cuidado ascendente, a la vez que los niveles de acuerdo disminuyen a medida que la posibilidad de este cuidado es más palpable, descenso que se acentúa en el tramo de edad de 55 a 64 años $(44,9 \%)$.

La percepción de obligatoriedad de cuidar a largo plazo a progenitores aumenta con características como la mayor religiosidad (60,9\%), que prescribe la obligatoriedad de honrar al padre y a la madre; el nacimiento fuera de España (72\%), en particular en Latinoamérica y Rumanía, lo que remite a diferencias culturales; la viudedad (63,5\%), que podría relacionarse con una previsible - y, en función de la edad, quizá cercana- necesidad de recibir cuidados; la convivencia con el padre o la madre (66\%), situación que podría asociarse, en edades jóvenes, a la distancia con la que se contempla el cuidado o, en edades más avanzadas, a una validación subjetiva del propio desempeño cotidiano de dicho cuidado; y, por último, la valoración y enseñanza familiar de valores tradicionales — como la fe religiosa (67,7\%), la abnegación ${ }^{12}$ (62,5\%) y el trabajo duro (58,9\%)—, necesarios y/o acordes con el trabajo que supone la prestación continuada de cuidados a ascendientes.

${ }^{12}$ En este contexto, la fe religiosa y la abnegación son valores asociados culturalmente a lo femenino. 
Cuidado, valores y género | I. Aristegui Fradua, U. Beloki Marañon, R. Royo Prieto, M. a Silvestre Cabrera

Tabla 6. Grado de acuerdo con la afirmación «Es obligación del hijo proporcionar cuidado a largo plazo para los padres.» \% horizontales. España 2018

\begin{tabular}{|c|c|c|c|c|c|c|}
\hline & $\begin{array}{l}\text { Muy de } \\
\text { acuerdo }\end{array}$ & Acuerdo & $\begin{array}{c}\text { Ni de } \\
\text { Acuerdo/ } \\
\text { Ni en } \\
\text { Desacuerdo }\end{array}$ & Desacuerdo & $\begin{array}{c}\text { Muy en } \\
\text { Desacuerdo }\end{array}$ & $\mathrm{NS} / \mathrm{NC}$ \\
\hline TOTAL & 15,3 & 36,8 & 22,0 & 17,7 & 6,8 & 1,4 \\
\hline \multicolumn{7}{|l|}{ Persona religiosa } \\
\hline SÍ & 20,6 & 40,3 & 17,6 & 14,3 & 6,4 & 7,2 \\
\hline NO & 9,7 & 31,1 & 29,2 & 20,4 & 7,6 & 1,9 \\
\hline \multicolumn{7}{|l|}{ Edad } \\
\hline $18-24$ & 23,6 & 39,3 & 18.0 & 14,6 & 1,1 & 3,4 \\
\hline $25-34$ & 13,8 & 41,9 & 22,2 & 12,6 & 9.0 & 0,6 \\
\hline $35-44$ & 15,3 & 32,9 & 19,4 & 22,1 & 8,6 & 1,9 \\
\hline $45-54$ & 9,9 & 37,5 & 21,7 & 20,9 & 8,7 & 1,2 \\
\hline $55-64$ & 11,4 & 33,5 & 23,8 & 22,7 & 8,1 & 0,5 \\
\hline $65 y+$ & 20,6 & 37,5 & 24,3 & 12,2 & 3,7 & 1,7 \\
\hline \multicolumn{7}{|l|}{ Lugar nacimiento } \\
\hline España & 14,0 & 35,4 & 23,4 & 18,5 & 7,4 & 1,4 \\
\hline Otros* & 24,7 & 47,3 & 12,3 & 11,6 & 2,7 & 1,4 \\
\hline \multicolumn{7}{|l|}{$\begin{array}{l}\text { Vive con los dos } \\
\text { o un padre/madre }\end{array}$} \\
\hline No & 14,7 & 36,0 & 22,6 & 18,3 & 7,0 & 1,4 \\
\hline $\mathrm{Si}$ & 23,0 & 43,0 & 17,0 & 11,0 & 5,0 & 1,0 \\
\hline \multicolumn{7}{|l|}{ Estado Civil } \\
\hline Casado/a & 12,9 & 35,8 & 22,1 & 19.9 & 7,5 & 1,9 \\
\hline Pareja de Hecho & 15,8 & 40,4 & 12,3 & 21,1 & 8,8 & 1,8 \\
\hline Viudo/a & 28,7 & 34,8 & 24,3 & 9,6 & 1,7 & 0,9 \\
\hline Separado/a & 17,4 & 29,0 & 18,8 & 24,6 & 10,1 & 0,0 \\
\hline \multicolumn{7}{|c|}{$\begin{array}{c}\text { Valores a aprender } \\
\text { en casa }\end{array}$} \\
\hline Responsabilidad & 15,9 & 36,2 & 22,4 & 17,0 & 7,0 & 1,5 \\
\hline Fe religiosa & 23,8 & 43,9 & 13,2 & 14,8 & 3,7 & 0,5 \\
\hline Abnegación & 23,2 & 39,3 & 25,0 & 8,9 & 1,8 & 1,8 \\
\hline Obediencia & 14,8 & 41,2 & 22,0 & 16,4 & 4,0 & 1,6 \\
\hline Trabajo duro & 16,3 & 42,6 & 18,3 & 16,6 & 4,5 & 1,7 \\
\hline
\end{tabular}

* En Otros (sobre todo Latinoamérica y Rumanía).

Fuente: Elaboración propia a partir de la base de datos de la EVS 2018. 


\section{CONCLUSIONES Y FUTURAS LÍNEAS DE INVESTIGACIÓN}

Este artículo realiza un primer análisis descriptivo de los recientes datos de la Encuesta Europea de Valores en su aplicación en España. La intención del artículo es iniciar una reflexión en torno a la prevalencia, o no, de una división sexual del trabajo en el ámbito del cuidado, tanto a menores como a personas mayores. Se trata de analizar si conviven nuevos roles laborales con viejos roles domésticos en la construcción del papel social de las mujeres. En este sentido, podemos afirmar que la extendida oposición a la otrora rígida división de roles familiares no implica que el referente doméstico-maternal haya desaparecido totalmente del imaginario de la sociedad española. Viejos y nuevos valores sobre el rol de las mujeres en la familia coexisten y compiten entre sí, si bien, como hemos visto, se distribuyen de forma heterogénea entre la población, en función del género y la edad.

Hemos observado que cuando hablamos de la crianza de la prole, las mujeres mayores de 65 años (43\%) y las mujeres de entre 35 y 44 años (29\%) son las que más cuestionan el desempeño de la maternidad cuando esta trata de compaginarse con un empleo a tiempo completo, en el sentido de que son las que más opinan que si una mujer trabaja a tiempo completo, la unidad familiar sufre. Nos interesa centrarnos en ese tercio de mujeres en la treintena que opinan de esa manera. No debemos pasar por alto que, según el INE, la edad media a la que una mujer en España tiene su primer hijo está en 31 años, por lo que deberíamos analizar con más profundidad esta afirmación en esa cohorte de edad, puesto que bien puede estar reflejando las dificultades de conciliar la vida personal, familiar y laboral de muchas madres, o bien puede estar demandando nuevas o viejas formas de ejercer la maternidad.

Por su parte, cuando hemos analizado los valores relacionados con la percepción de responsabilidad con el cuidado ascendente nos hemos encontrado con la persistencia de un modelo de sociedad familista donde prevalece la asunción de dicha responsabilidad y en el que, en el plano valorativo, hemos observado que los valores religiosos y la atribución de valor a la abnegación en la educación de los hijos e hijas inciden en una mayor asunción de responsabilidad en el cuidado de las personas mayores. También se ha revelado significativa la edad, sobre todo entre las personas más mayores, bien sea porque necesitan del cuidado, bien sea porque ya están cuidando. En este punto, merecerá la pena profundizar en el perfil del 22\% de la ciudadanía que no sabe o no responde a esta cuestión, porque podría estar ocultando la prevalencia de una actitud intergeneracional menos solidaria o bien cuestionando una obligación moral asumida de manera apriorística. Por último, no queremos dejar de mencionar en estas conclusiones la distancia que parece establecerse entre los valores o el discurso socialmente establecido y el ejercicio efectivo del cuidado, puesto que en contraposición a la feminización del cuidado (tanto de la prole, pero sobre todo de las personas mayores, de las personas con discapacidad o enfermas), no se recoge una significación estadística en los valores manifestados por mujeres y hombres. Quizá merezca la pena profundizar en la existencia o no de conciencia de género, sobre todo entre las mujeres que cuidan. 


\section{REFERENCIAS BIBLIOGRÁFICAS}

AlBERDI, Inés (dir.) (1999). La nueva familia española, Madrid: Taurus.

Barnett, Rosalind C. y Baruch, Grace K. (1988). «Correlates of Fathers' Participation in Family Work», en P. Bronstein y C. P. Cowan (eds.). Fatherhood Today. Men's Changing Role in the Family, United States of America: John Wiley \& Sons, pp. 66-78.

Beck, Ulrich y Beck-Gernsheim, Elisabeth. (2003). La individualización. El individualismo institucionalizado y sus consecuencias sociales y políticas, Barcelona: Paidós, Estado y Sociedad.

CAstells, Manuel (1997). La era de la información: Economía, sociedad y cultura. El poder de la identidad, Madrid: Alianza, Vol. II.

Carrasco, Cristina (Ed.) (2003). «¿Conciliación? No, gracias. Hacia una nueva organización social», en M. I. Amoroso, A. Bosch, C. Carrasco, H. Fernández y N. Moreno. Malabaristas de la vida. Mujeres, tiempos y trabajos, Barcelona: Icaria, pp. 27-51.

Carrasco, Cristina; Mayordomo, Maribel; Domínguez, Màrius y Alabart, Anna (2004). Trabajo con mirada de mujer. Propuesta de una encuesta de población no androcéntrica, Madrid: Consejo Económico y Social, Colección Estudios.

Coltrane, Scott (1996). Family Man, Fatherhood, Housework, and Gender Equity, New York: Oxford University Press.

-(1998). Gender and Families, California: A Sage publication/Pine Forge Press Series, The Gender Lens.

-(2000). «Research on Household Labor: Modeling and Measuring the Social Embeddedness of Routine Family Work», Journal of Marriage and the Family, n. ${ }^{\circ} 62$, pp. 1208-1233.

Chorodow, Nancy y Contratto, Susan (1982). "The Fantasy of the Perfect Mother», en B. Thorne (ed.). Rethinking the Family. Some feminist Questions, New York: Longman.

Cid López, Rosa María (2002). «La maternidad y la figura de la madre en la Roma antigua», en A. I. Blanco García, B. Doménech Delgado, M. S. López Rodríguez y R. Marcos Santiago (Coords.). Nuevas visiones de la maternidad, León: Universidad de León. Secretariado de publicaciones y medios audiovisuales, pp. 11-49.

Coontz, Stephanie (2005). Marriage, a History. From Obedience to Intimacy or How Love Conquered Marriage, New York: Viking Penguin. 
Cuidado, valores y género | I. Aristegui Fradua, U. Beloki Marañon, R. Royo Prieto, M.a Silvestre Cabrera

DIAZ, Capitolina y SIMÓ NOGUERA, Carles X. (2016). Brecha salarial y brecha de cuidados, Valencia: Tirant lo Blanch.

Di Quinzio, Patrice (1999). The Impossibility of Motherhood: Feminism, Individualism and The Problem of Mothering, ReinoUnido: Routledge.Durán Heras, María Ángeles (1978). El ama de casa. Crítica política de la economía doméstica, Madrid: Zero Zyx.

Donapetry, María (2002). "Cinematernidad: Todo sobre mi madre y Solas», en A. I. Blanco García; B. Doménech Delgado, M. S. López Rodríguez y R. Marcos Santiago (Coords.). Nuevas visiones de la maternidad, León: Universidad de León. Secretariado de Publicaciones y Medios Audiovisuales, pp. 51-71.

Dones I Treballs, Grupo (2003). «Introducción: Repasar desde el feminismo los tiempos y trabajos en la vida cotidiana», en M. I. Amoroso, A. Bosch, C. Carrasco, H. Fernández y N. Moreno. Malabaristas de la vida. Mujeres, tiempos y trabajos, Barcelona: Icaria, pp. 13-25.

ERICKson, Rebecca J. (2005). «Why Emotion Work Matters: Sex, Gender, and the Division of Household Labor», Journal of Marriage and Family, n. ${ }^{\circ}$ 67, pp. 337-351.

Folbre, Nancy y Bittman, Michael (Eds.) (2004). Family Time. The Social Organization of Care, Padstow: Routledge.

GidDENS, Anthony (2002). Sociología, Madrid: Alianza.

Hochschild, Arlie Russell y Machung, Anne (2003). The Second Shift, New York: Penguin Books.

Instituto Nacional de Estadística (INE) (2018). Mujeres y hombres en España, enhttp://www. ine.es/ss/Satellite?L=0\&c=INEPublicacion_C\&cid=1259924822888\&p=12547351106 72\&pagename=ProductosYServicios\%2FPYSLayout\&param1=PYSDetalleGratuitas (consultado el 20 de agosto de 2018).

Instituto Nacional de Estadística (INE) (2018).Edad media a la maternidad por orden de nacimiento según nacionalidad de la madre, en http://www.ine.es/jaxiT3/Datos. htm?t=1579, (consultado el 23 de noviembre de 2018)

Instituto Nacional de Estadística (INE) y Eurostat (2017). La vida de las mujeres y los hombres en Europa. Un retrato estadístico, enhttp://www.ine.es/prodyser/myhue17/bloc-3d. html?lang=es, (consultado el 21 de agosto de 2018)

Instituto Nacional de Seguridad e Higiene en el Trabajo (INSHT) (2017). Encuesta nacional de condiciones de trabajo 6. ${ }^{a}$ EWCS-España, en http://www.insht.es/InshtWeb/ 
Contenidos/Documentacion/FICHAS\%20DE\%20PUBLICACIONES/EN\%20CATALOGO/ GENERALIDAD/ENCT\%202015.pdf (Consultado el 19 de agosto de 2018).

Gorz, André (1997). Metamorfosis del trabajo. Búsqueda de sentido. Crítica de la razón económica, Madrid: Sistema.

LAGARDE, Marcela (1996). Género y feminismo: desarrollo humano y democracia, Madrid: Horas y Horas, Cuadernos Inacabados, n. ${ }^{\circ} 25$.

LEonARDo, Jon et al. (2007). Los cuidados informales en la Comunidad Autónoma de Euskadi: las necesidades de las personas cuidadoras, Vitoria-Gasteiz: Servicio Central de Publicaciones del Gobierno Vasco, Documentos de Bienestar Social. Volumen 72.

MANDER, Rosemary (2004). Men and Maternity, Padstow, Cornwall: Routledge.

McDougal, Mary L. (1984). «Mujeres trabajadoras durante la revolución industrial, 17801914», en M. NASH (Ed.). Presencia y protagonismo. Aspectos de la historia de la mujer, Barcelona: Serbal, pp. 91-119.

MeilLandwerlin, Gerardo (1999). La postmodernización de la familia española, Madrid: Acento.

Moss, Peter (1995). Father Figures. Fathers in the families of the 1990s, Edinburgh: HMSO.

OsBorne, Raquel (1993). La construcción sexual de la realidad, Madrid: Cátedra.

Papí Gálvez, Natalia y Frau Llinares, M. a José (2005). «La conciliación del empleo y del hogar: respuesta y reflejo de una organización del trabajo construida desde la institución del género», Reis, n. ${ }^{\circ}$ 110, pp. 149-171.

Parke, Ross D. (2000). «Father Involvement: A Developmental Psychological Perspective», en H. E. Peters, G. W. Peterson, S. K. Steinmetz y R. D. Day (eds.). Fatherhood: Research, Interventions and Policies, United States of America: The Haworth Press, pp. 43-58.

Peterson, G. W. y Steinmetz, S. K. (2000). «The Diversity of Fatherhood. Change, Constancy and Contradiction», en H. E. Peters, G. W. Peterson, S. K. Steinmetz y R. D. Day (Eds.). Fatherhood: Research, Interventions and Policies, United States of America: The Haworth Press, pp. 315-322.

Royo, RaQuel (2011). Maternidad, paternidad y conciliación en la CAE. ¿Es el trabajo familiar un trabajo de mujeres?, Bilbao: Universidad de Deusto. 
Cuidado, valores y género | I. Aristegui Fradua, U. Beloki Marañon, R. Royo Prieto, M.a Silvestre Cabrera

ScotT, Joan Wallach y Tilly, Louise A. (1984). «El trabajo de la mujer y la familia en Europa durante el siglo xIx», en M. NAsH (ed.). Presencia y protagonismo. Aspectos de la historia de la mujer, Barcelona: Serbal, pp. 51-90.

Todaro, Rosalba y Rodríguez, Regina (eds.) (2001). El género en la economía, Santiago, Chile: Isis Internacional, Ediciones de las Mujeres, n. 32.

Torns Martín, Teresa (2005). «De la imposible conciliación a los permanentes malos arreglos», Cuadernos de Relaciones Laborales, 23, n. ${ }^{0}$ 1, pp. 15-33.

Swigart, Jane (1991). The Myth of Bad Mother, New York: Doubleday. 